\title{
A Computational Discriminability Analysis on Twin Fingerprints
}

\author{
Yu Liu, Sargur N. Srihari \\ Department of Computer Science and Engineering, \\ University at Buffalo, The State University of New York, Buffalo NY, USA \\ $\{$ yl73, srihari\}@buffalo.edu
}

\begin{abstract}
Sharing similar genetic traits makes the investigation of twins an important study in forensics and biometrics. Fingerprints are one of the most commonly found types of forensic evidence. The similarity between twins' prints is critical establish to the reliability of fingerprint identification. We present a quantitative analysis of the discriminability of twin fingerprints on a new data set (227 pairs of identical twins and fraternal twins) using both level 1 and level 2 features. Although the patterns of minutiae among twins are more similar than in the general population, the similarity of fingerprints of twins is significantly different from that between genuine prints of the same finger. Twins fingerprints are discriminable with a $1.5 \% \sim 1.7 \%$ higher EER than non-twins.
\end{abstract}

\section{Introduction}

The studies of twins ramify into genetic [2], physiological [3], biochemical [1] and sociological aspects. There are two types of twins, dizygotic twins (commonly known as fraternal twins) and monozygotic twins, frequently referred to as identical twins. Fraternal twins occur when two fertilized eggs are implanted in the uterine wall at the same time. Identical twins develop when a single fertilized egg splits in two embryos. Because sharing a single zygote, identical twin individuals will have the same genetic makeup. Although there are lot of traits about twins that are not entirely genetic characteristics, twins still show high similarity in appearance, behavior, traits such as fingerprints, speech patterns and handwriting. Genetic and environmental similarities of twins allow studies such as the effectiveness of drugs, presence of psychological traits, etc. By examining the degree to which twins are differentiated, a study may determine the extent to which a particular trait is influenced by genetics or by the environment.

In forensics, questions concerned are how do the inherited and acquired traits of twins differ from those of singletons? How do the traits of identical twins differ from those of non-identical twins? By what means can twins be distinguished from other singletons? While having identical DNA makes it difficult for forensic scientists to distinguish between DNA from blood samples of identical twins. Few twin studies have been carried out in any modality due to the lack of sufficient data. Such studies are important since any modality needs to be evaluated in conditions under which the possibility of error is maximum, i.e., the worst-case 
scenario. Satisfactory performance with twins strengthens the reliability of the method. It also establishes the degree of individuality of the particular trait. Such an individuality measure is relevant from the viewpoint of Daubert challenges in forensic testimony [6].

A forensic phonetic investigation on the speech patterns of identical and nonidentical twins has indicated that they could be discriminated using Bayesian Likelihood Ratios [5]. A significant number of twin pairs (206) have been studied for handwriting [8]. These samples were processed with features extracted and conclusions drawn by comparing verification performances with twins and non-twins. In that study the conclusion was that twins are discriminable but less so than an arbitrary pair of individuals. An examination on palmprints generated from the same genetic information was carried out [7] and an automatic parmprint identification algorithm is provided to distinguish identical twins' palmprints. The study showed a significant correlation within identical twin matching (prints generated from the same genetic information).

The question for fingerprint is whether there exists a higher degree of similarity between individuals who are twins rather than when the individuals are not twins. The goal is to determine whether the fingerprints of twins are more similar to each other than in the case of the general population. A study on fingerprint of identical twins has been previously reported with a small data set of 94 pairs of index fingers [10]. Using a state-of-the-art fingerprint verification system it was concluded that identical twins are discriminable with slightly lower accuracy than non-twins. The marginal degradation in performance may be attributed to the dependence of minutiae distribution on fingerprint class. An earlier study [11] made use of fingerprints of 196 pairs of twins. In that study 196 comparisons of level 1 classification were made and when there was a match, a ridge count comparison was made. Level 2 (minutiae) comparison included only 107 pairs corresponding to identical twin fingerprints.

In our previous twins' fingerprints study [12], we had compared the distribution of twins fingerprint classes to general population. The inferences that twins are different from genuines were made by statistic test of the similarity of matching score distribution of twins and that of the geunine distribution using a twins' database collected in 2003. In this study, we quantitively analyze the similarity of identcial twins and fraternal twins using both level 1 and level 2 features on a later Twins' data set collected in 2007.

The rest of this paper is organized as follows. Section 2 introduces the new Twins data set used in this study. Section 3 presents our experiment results and analysis. Section 4 gives summary and conclusions.

\section{Twins Data Set}

The data set used in this study consisted of friction ridge patterns of over five hundred pairs of twins. This data set was collected by the International Association for Identification (IAI) at a twins festival held in Twinsburg, Ohio in August 2007. The friction ridge images of 477 individuals including 227 sets of 
twins(188 sets of identical twins and 39 sets of fraternal twins) and one sets of triplets. For each individual there are ten fingerprints, thus making available 2,270 pairs of twin's fingers. In addition there are palm prints, DNA samples and latent prints collected, but not used in this study. In our earlier study [12], similar twins' data set used are collected by IAI in 2003. The electronic capture of fingerprints were scanned by Smith Heiman scanner and processed with Cogent System, Inc. Software. images were obtained at 500 dpi. The 2007 Twins data set is of a higher quality than the 2003 Twins data set.

A meta-data table accompanying each folder of fingerprint images gives the demographic information for the individual, code for the individual and a pointer to his/her twin. The demographic information consists of age, gender, hair color, racial characteristics, whether twins are identical or fraternal, and handedness (left/right). The distribution of ages of the twins shows that the twins in the study are predominantly in their adolescent years. Thus the quality of their fingerprints can be expected to be good.

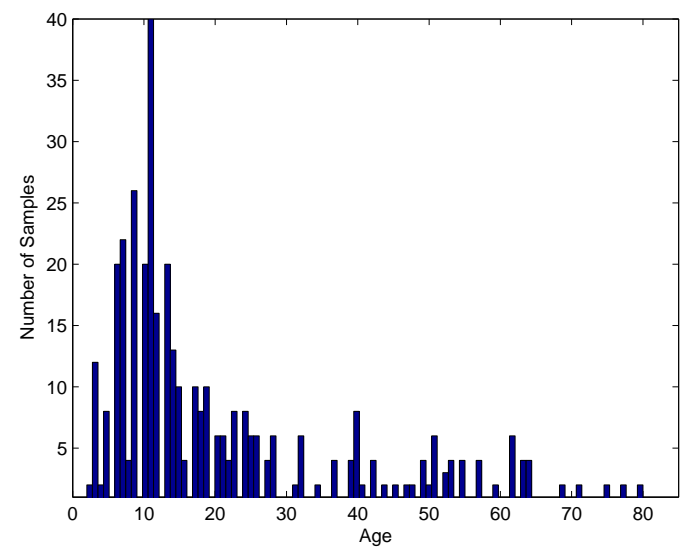

Fig. 1. Distribution of twins' ages in database: a predominance of younger ages is observed.

\section{Experiment Results}

Friction ridge patterns contained in fingerprints can be analyzed at several levels of features. Level 1 features correspond to visually observable characteristics commonly used in fingerprint classification, i.e., arch, loop, whorl are the most common fingerprint ridge flow types. Level 2 features correspond to minutiae, which are primarily points corresponding to ridge endings and ridge bifurcations. Level 3 features include pores within ridges, ridge widths, and shapes. The analysis reported here was done using level 1 and level 2 features. 


\subsection{Level 1 Study}

The first study was to determine the similarities at Level 1 or fingerprint classes. A number of studies have shown that there are correlation in the fingerprint class (level 1 features) of identical twin fingers [9]. Correlation based on other generic attributes of the fingerprint such as ridge count, ridge width, ridge separation, and ridge depth has also been found to be significant in identical twins. In our study, the twins fingerprint images are labeled according to one of six categories: arch, tented arch, right loop, left loop, whorl and twin loop [13]. The overall distribution of the six level 1 features provides an indication of how frequently each class is encountered in the Twins database, i.e., arch (5\%), tented arch (3\%), left loop (32\%), right loop (34\%), whorl (20\%) and twin loop (6\%).

The analysis consisted of determining as to how often the prints of the same finger in a pair of twins matched and a comparison with the case of non-twins. For twins, we count the total number of finger pairs that belong to the same fingerprint class. Among the 227 pairs of twins, the percentage of times twins had the same class label for a given finger was $62.78 \%$. If identical twins is considered, the percentage of same level 1 was $66.71 \%$ as against $46.41 \%$ for fraternal twins. For non-twins, if we compute the probability that any two fingerprints share the same class label in the Twin' data set is as $p_{\text {arch }}^{2}+p_{\text {tented arch }}^{2}+p_{\text {left loop }}^{2}+$ $p_{\text {right loop }}^{2}+p_{\text {whorl }}^{2}+p_{\text {twin loop }}^{2}$, that is 0.265 . Then the probability of any two person who are not twins having the same level 1 type is no more than $26.5 \%$, is much lower than the probability of twins' match. In [10], the probability that two randomly picked fingerprints that have the same class label is computed to be $27.18 \%$ according to five major fingerprint classes in the index finger based on an unpublished FBI report using database of 22, 000, 000 human-classified fingerprints. Thus we can conclude that twins are more than twice as likely as non-twins in matching level 1 features. Figure 2 shows an examples of exact same class labels of 10 pairs of prints belonged to one identical twins.

Our results demonstrate a significant correlation in level 1 features of twins, especially identical twins. However, level 1 features are used only as a coarse method of eliminating candidates from a large database as in automatic fingerprint identification systems (AFIS). It has no implication on the discriminability of twins since level 1 features are not used in fingerprint identification.

\subsection{Level 2 Study}

The most important part of the study from the point of view of identification involves level 2 features since they are what are used by AFIS systems in fingerprint matching. Level 2 features consist of minutiae which are either ridge endings or ridge bifurcations. Each minutia is represented by a 3 -tuple $(x, y, \theta)$ representing its position and orientation in the fingerprint image. The question to be examined is as to whether the fingerprints match when minutiae are used as features. To quantitatively measure similarity, we use an AFIS type algorithm that extracts minutiae and obtains a score from the comparison. The MIN-DTCT algorithm for detecting minutiae and the Bozorth matcher [14], which provides 

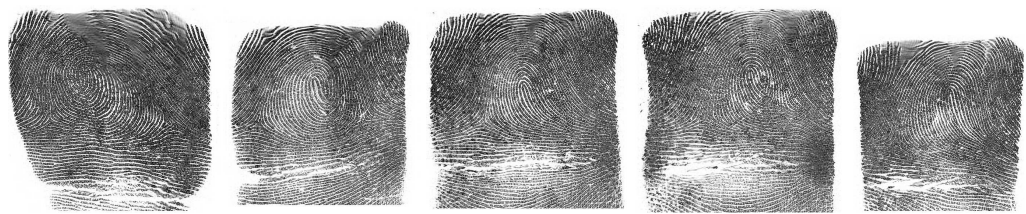

(a)
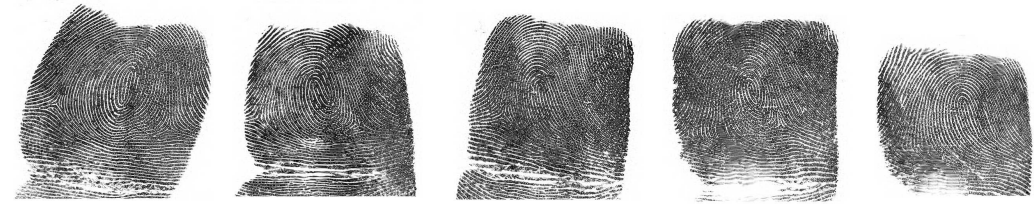

(b)
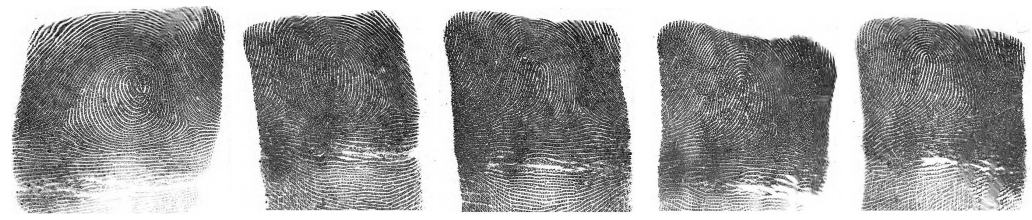

(c)
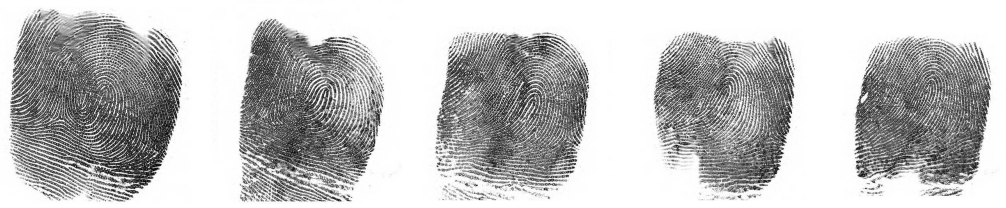

(d)

Fig. 2. Fingerprint examples for an identical twin. (a) and (c) are 10 fingerprint images of an individual A, (b) and (d) are 10 fingerprint images of the corresponding twin B. The twins have exactly identical fingerprint classes for both hands. (Class labels are: (left-hand) whorl, twin loop, right loop, whorl, right loop; (right-hand) twin loop, whorl, left loop, left loop, left loop) 
a score for the match of a pair of fingerprints, both of which are available from NIST, was used to compare fingerprint pairs. The Bozorth score is typically in the range of 0-50 for impostor scores and can be as high as 350 for genuine (see Figure 3). Other AFIS algorithms have similar scores but have different ranges. Figure 4 shows three fingerprints images and extracted minutiae superimposed on the corresponding skeleton prints. Two of them are an identical twin and the other is an unrelated individual. The matching score between the twin is slightly higher than that between non-twin, but both imposter scores are less than 50 .

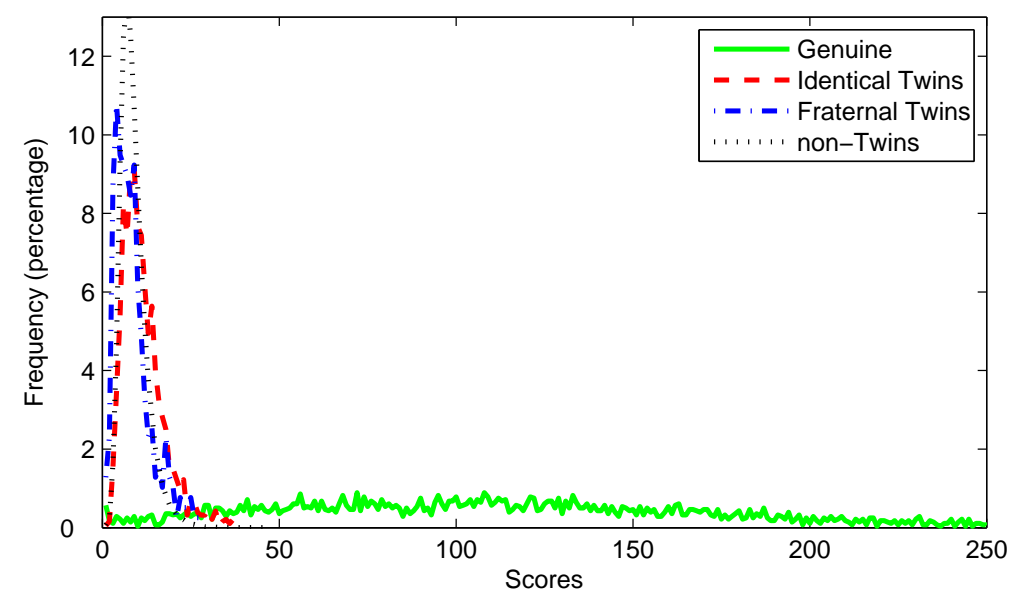

Fig. 3. Matching score distributions.(The following distributions were obtained: nonTwin: An individual's fingerprint was matched with the corresponding fingerprint of all other people who were not his/her twin. Identical: The fingerprint of an individual was matched with the corresponding fingerprint of his/her identical twin(twin-twin match). Fraternal: The fingerprint of an individual was matched with the corresponding fingerprint of his/her fraternal twin(twin-twin match). Genuine: Fingerprints Pairs in FVC2002 DB1 belonging to a same finger were compared against each other.)

All 227 pairs of twins was used to carry out the experiments. The fingerprints are rolled fingerprints with 10 prints(corresponding to 10 fingers) per person. For twin-twin match, the imposter score distribution is obtained from the total 2, 270 comparisons. Out of these 780 were prints of fraternal twins and the remaining 1,880 were those of identical twins. For non-twin imposter match, we compare fingerprints among 227 individuals without their corresponding twins. An individual's fingerprints are matched with the corresponding fingerprints of all other people who is unrelated to him/her. The total number of comparisons was $256,510((10 \times 227 \times 226) / 2)$. In order to get a score distribution of genuine match (pairs of fingerprints that belong to the same finger were compared against each other), we used FVC2002 DB1 data set, due to the lack of multiple rolled 


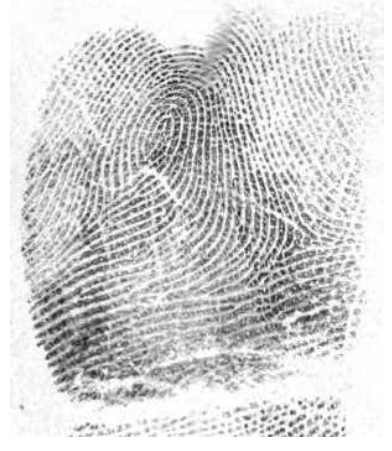

(a)

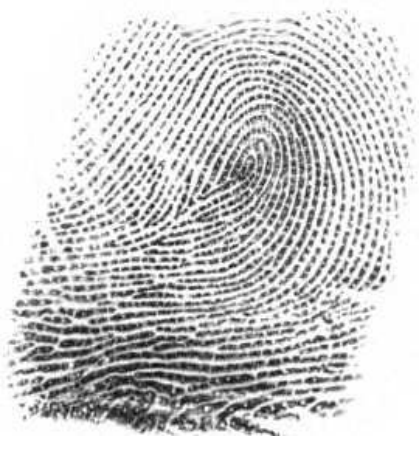

(c)

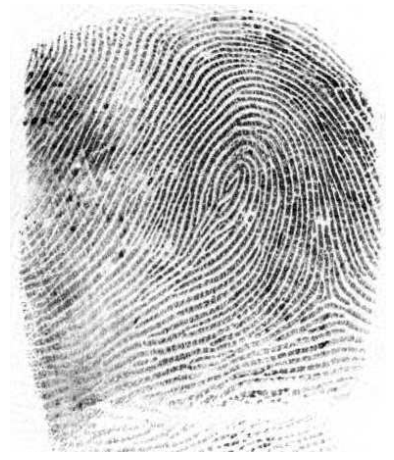

(e)

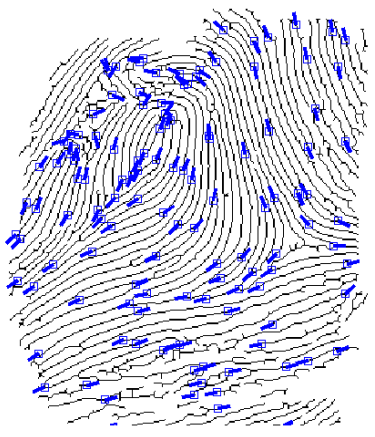

(b)

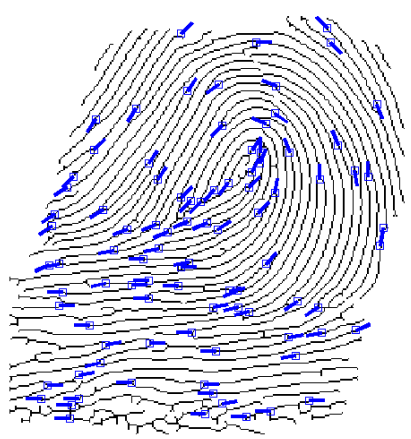

(d)

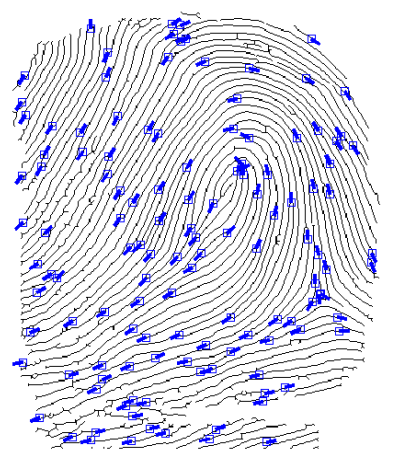

(f)

Fig. 4. Similar fingerprint images with extrated minutiae. (a) and (c) are index fingerprint images of an identical twin, (e) is an index fingerprint image of another unrelated person. (b), (d), (f) are the corresponding minutiae extracted (with their locations and orientations indicated). The matching score between (a) and (c) is 47; while the matching score between (a) and (e) is 7 . 
fingerprint samples of the same finger in the Twins' data set. A total of 100 fingers with 8 samples of each finger constituting a total of 800 prints were present in the database. The genuine scores are obtained by comparing each fingerprint with the rest of the 7 impressions resulting a $2800(100 \times(8 \times 7) / 2$ matchings. There were also obtained as livescan image at $500 \mathrm{dpi}$, similar to the Twins' data set. Figure 3 shows these distributions. A slight shift in the twins imposter distribution in comparison to the non-twins imposter distribution can be observed, which gives an impression that distinguishing twins are slightly harder than distinguishing any two random people by matching their fingerprints since false positive rate is increased. Besides, the distribution of matching score of identical twins lie a little bit right to the that of fraternal twins indicating a slightly increase of score on average level for matching identical twin.

The Kolmogorov-Smirnov(KS) Goodness-Of-Fit Test By statistically comparing probability of similarity among the score distributions for twins, nontwin and genuine matching, we need to answer a question: "Can we disprove, with a certain required level of significance, the null hypothesis that the two distributions are drawn from the same population?"[15]. The test first obtains the cumulative distribution functions of each of the distributions to be compared, and then computes the statistic, $D$, as the maximum value of the absolute difference between two of the cumulative distribution functions to be compared. What makes the KS statistic useful is that the distribution in the case of the null

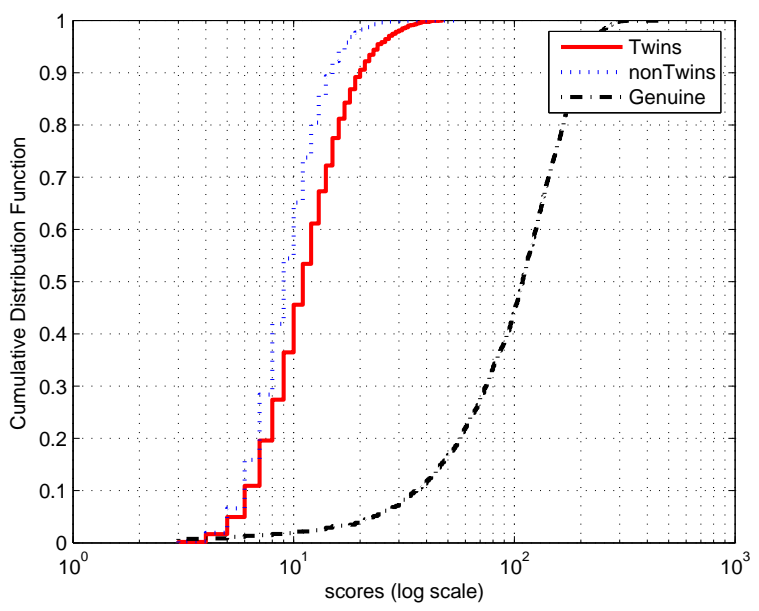

Fig. 5. Culmulative distribtuion function for Kolmogorov-Smirnov test

hypothesis (data sets drawn from the same distribution) can be calculated, at least useful approximation, thus giving the significance of any observed nonzero 
value of $D$. For example, if comparing twins with non-twin, the KS statistic $D$ is given by $D=\max _{-\infty<x<\infty}\left|S_{\text {twins }}(x)-S_{\text {non-twin }}(x)\right|$ (see Figure 5), where $x$ denotes the matching score and $S_{\text {twins }}(x)$ and $S_{\text {non-twin }}(x)$ are cumulative distribution functions for twins and non-twin. The significance level of an observed value of $D$ is given approximately [15].

We computed the significance of $D$ between any two distributions shown in Figure 5. The resulting p-value of the indicates a significance level that the null hypothesis is accepted. For both twin-genuine and nontwin-genuine comparisons, the computed probability of any observed nonzero value of $\mathrm{D}$ is 0 . For twin-nontwin comparison, the p-value is slightly larger than 0 (5.9957e-082) which indicates a $99.99 \%$ confidence level of rejecting the null hypothesis. The conclusion can be drawn as there is a significantly different between matching scores distribution of twins and that of non-twins. This distribution difference can also be observed from Figure 5. Moreover, the p-value of comparing identical twins and fraternal twins is found to be 0.012. Although it is not strong enough to reject null hypothesis if the $1 \%$ significance level is considered. The result still indicates that identical twins share more similarity in the level 2 feature than the fraternal twins. As a whole, the similarity of fingerprints of twins is different from that between genuine prints of the same finger.

Receiver Operating Characteristics(ROC) Analysis In a biometric verification system, the system decision is normally regulated by a threshold $t$. The scores provided by the matcher can be thresholded to provide a hard decision of being the same or different.

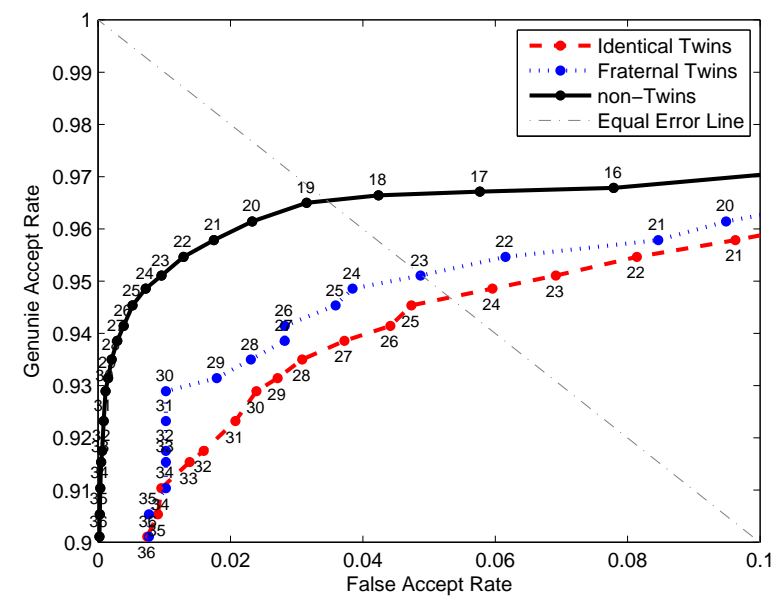

Fig. 6. ROC curves of twins and non-twin matching. Operating points(thresholds) are also shown along the curves. 
The ROC curves are in Figure 6 for matching twins and non-twins, as well as the system decision thresholds shown along the curve. The ROC curve of the matching twin pairs (including identical twins and fraternal twins) are lower than that of non-twin pairs. There is a tradeoff between FAR and FRR that both depend on the choice of threshold. The equal error rate (EER) with the corresponding operation threshold point for all three cases is shown in Table 1. The average error rate for matching identical twins is roughly $1.7 \%$ higher than non-twin matching and the average matching error for fraternal twins is $1.5 \%$ higher than non-twin matching if the operating point is selected based on EER.

Table 1. False Acceptance Rate, False Rejection Rate and Average Error Rate with different thresholds $(19 \sim 25)$ for twin and non-twin matching in the Twins data set (the Equal Error Rate is underlined).

\begin{tabular}{|c|c|c|c|c|c|c|c|}
\hline \multirow[b]{2}{*}{ Threshold } & \multirow[b]{2}{*}{$\begin{array}{l}\text { FRR } \\
(\%)\end{array}$} & \multicolumn{2}{|c|}{ non-twin } & \multicolumn{2}{|c|}{ fraternal } & \multicolumn{2}{|c|}{ identical } \\
\hline & & $\begin{array}{l}\text { FAR } \\
(\%)\end{array}$ & $\begin{array}{c}\text { AER } \\
(\%)\end{array}$ & $\begin{array}{c}\text { FAR } \\
(\%)\end{array}$ & $\begin{array}{c}\text { AER } \\
(\%)\end{array}$ & $\begin{array}{c}\text { FAR } \\
(\%)\end{array}$ & $\begin{array}{c}\text { AER } \\
(\%)\end{array}$ \\
\hline 19 & 3.5 & 3.15 & 3.33 & 11.03 & 7.26 & 13.62 & 8.56 \\
\hline 20 & 3.86 & 2.33 & 3.09 & 9.49 & 6.67 & 11.12 & 7.49 \\
\hline 21 & 4.21 & 1.75 & 2.98 & 8.46 & 6.34 & 9.63 & 6.92 \\
\hline 22 & 4.54 & 1.29 & 2.91 & 6.15 & 5.34 & 8.14 & 6.34 \\
\hline 23 & 4.89 & 0.96 & 2.93 & 4.87 & 4.88 & 6.91 & 5.90 \\
\hline 24 & 5.14 & 0.72 & 2.93 & 3.85 & 4.49 & 5.96 & 5.55 \\
\hline 25 & 5.46 & 0.52 & 2.99 & 3.59 & 4.53 & 4.73 & $\underline{5.09}$ \\
\hline
\end{tabular}

Because the matching score distribution for twins are slight closer to the genuine score distribution comparing to the non-twin matching scores, FAR is increased as a given threshold but the FRR remains unchanged (same threshold for different matching scenarios lie on a same horizontal line in Figure 6). Table 1 shows the FAR and FRR values with different thresholds ranging from 19 to 25 for non-twin and twin matching. The discriminability study of Twins data shows that for a given threshold at 25, at which a EER can be achieved and there is a chance of $5.46 \%$ that the same finger can be mistakenly rejected, a FAR of $0.52 \%$ for non-twin matching and a FAR of $4.73 \%$ for identical twins indicates fingerprints of twins are 9 times more likely than non-twin to be falsely accepted. While In cases of forensic applications such as criminal identification, where FRR is a major concern, by lowering the threshold to 19, a FAR of $3.15 \%$ for non-twin matching and a FAR of $13.62 \%$ for identical twins indicates fingerprints of twins are 4 times more likely to to be falsely accepted than unrelated people. For example, in a fingerprint identification system with 1 million nontwin individuals enrolled, 31500 people will be falsely accepted at the threshold of 19. If such system contains 500,000 identical twins fingerprint pairs, there would be 136000 people be falsely accepted. Figure 7 illustrates the ratio of false match (and false non-match) between twins and non-twins against the choice of system threshold. With the increase of the threshold, twin imposters are more 
likely to be falsely accepted than non-twin imposter. Besides, due to the genetically similarity between identical twins, they tend to be more hard to be distinguished than fraternal twins.

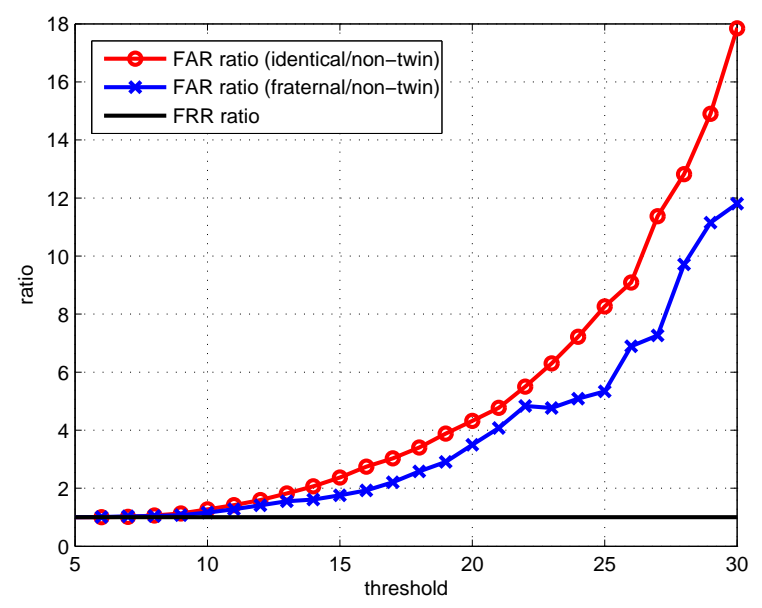

Fig. 7. Ratio between FAR and FRR for twins and non-twin matching

\section{Summary and Conclusion}

A study of the discriminability of the fingerprints of twins using a latest set of samples has been presented. Live scans and younger ages of the subjects ensured good quality prints thereby allowing the focus to be on the inherent individuality of fingerprints and one that was not affected by image quality issues. The similarities of the fingerprints of both identical twins and fraternal twins were studied using fingerprint features at levels 1 and 2. The level 1 results show that identical twins finger's have a significant correlation in fingerprint class leading to a higher probability of having the same type of ridge flow $(62.78 \%$ for all twins) than in the case of non-twins (26.5\%). Level 2 features were studied using a minutiae-based matching algorithm which provides a similarity score. Distributions of scores were compared using the Kolmogorov-Smirnov test. The system errors at different operating points are analyzed for the cases of twintwin match and non-twin matching. The statistical inferences from the level 2 study are: comparing to fingerprint imposters from random unrelated people, it is more difficult to distinguish fingerprints of twins. The EER for matching twins is roughly $1.5 \% \sim 1.7 \%$ higher than non-twin matching. However, the similarity of fingerprints of twins is significantly different from that between genuine prints of the same finger. Besides, due to the inherit similarity between identical twins, 
they can be distinguished by examine fingerprint with a slightly higher error rate than fraternal twins. This leads to a conclusion differing from the previous 2003 Twins data set.

Similar to the study on 2003 Twins data set, the implications are that there is more similarity between twin fingers than in the case of two arbitrary fingers, and that twins can be successfully discriminated using fingerprints.

\section{References}

1. Evans, A.; van Baal, G. C. M.; McCarron, P.; deLange, M.; Soerensen, T. I. A.; de Geus, E. J. C.; Kyvik, K.; Pedersen, N. L.; Spector, T. D.; Andrew, T.; Patterson, C.; Whitfield, J. B.; Zhu, G.; Martin, N. G.; Kaprio, J.; Boomsma, D. I.: The Genetics of Coronary Heart Disease: The Contribution of Twin Studies. Twin Research 2003, 6 (5), 432-441.

2. Rubocki, R.; McCue, B.; Duffy, K.; Shepard, K.; Shepherd, S.; Wisecarver, J.: Natural DNA Mixtures Generated in Fraternal Twins in Utero. J. For. Sci. 2001, 46 (1), 120-125.

3. Spitz, E.; Mountier, R.; Reed, T.; Busnel, M. C.; Marchaland, C.; Robertoux, P. L.; Carlier, M.: Comparative Diagnoses of Twin Zygosity by SSLP Variant Analysis, Questionnaire, and Dermatoglyphic Analysis. Behav. Genet. 1996, 26 (1), 55-63

4. Goldberg, S.; Perrotta, M.; Minde, K.; Corter, K.: Maternal Behaviour and Attachment in Low Birthweight Twins and Singletons. Child Dev. 1986, 57 (1), 34-46.

5. Deborah Loakes: A forensic phonetic investigation into the speech patterns of identical and non-identical twins. IJSLL vol 15.1, 2008, 97-100.

6. Daubert v Merrell Dow Pharmaceuticals, Inc. 509 U.S. 579, 113 S.Ct. 2786 (1993).

7. Adams Wai-kin Kong, David Zhang, Guangming Lu: A study of identical twins' palmprints for personal verification. Pattern Recognition. 39 2006, 2149-2156.

8. rihari, S. N.; Srinivasan, C. H.: On the Discriminability of the Handwriting of Twins. J. For. Sci. (Accepted for publication)

9. Jain, A. K.; Prabhakar, S.; Pankanti, S.: Twin Test: On Discriminability of Fingerprints. In Lecture Notes in Computer Science, Proceedings of the AVBPA International Conference, Halmstad, Sweden, June 6-8, 2001; Springer-Verlag: London, UK, 2001, pp 211-216.

10. Jain, A. K.; Prabhakar, S.; Pankanti, S.: On similarity of identical twin fingerprints. Pattern Recognition, 35, 2002, 2653-2663.

11. Lin, C. H.; Liu, J. H.; Osterburg, J. W.; Nicol, J. D.: Fingerprint Comparison 1: Similarity of Fingerprints. J. For. Sci. 1982, 27 (2), 290-304.

12. Srihari, S.N. Srinivasan, H. Fang, G.: Discriminability of Fingerprints of Twins. Journal of Forensic Identification. 2008, Vol. 58, 109-129.

13. Maltoni, D.; Maio, D.; Jain, A. K.; Prabhakar, S.: Handbook of Fingerprint Recognition. Springer: New York, 2003; p 176.

14. Garris, M. D.; Watson, C. I.; McCabe, R. M.; Wilson, C. L.: Users Guide to NIST Fingerprint Image Software (NFIS); NISTIR 6813; NIST, U.S. Dept. of Commerce: Gaithersburg, MD, 20

15. Press, W. H.; Flannery, B. P.; Teukolsky, S. A.; Vetterling, W. T.: Numerical Recipes in C: The Art of Scientific Computing, 2nd ed.; Cambridge University Press: Cambridge, MA, 1992. 